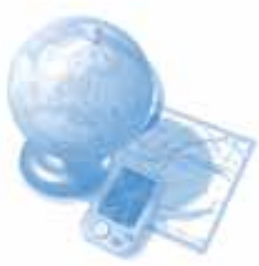

Michael Bridge*
Serena Corr
Sylvia Draper
Noelle Scully
School of Chemistry
Trinity College
Dublin 2
Ireland

*mbridge@tcd.ie

\section{Safe Labs for Science: an interactive approach to safety training}

\begin{abstract}
Safe working in Chemistry involves critical analysis, interactive thinking and the evolving application of risk assessment to procedure, reagent and environment. It also requires cooperation and teamwork. These are transferable skills that all scientists should possess, but safety training is too often presented to undergraduate students as a set of rules with little rationale offered to justify them. Attempts to go beyond a set of rules are frequently perceived by students and academics as tedious if not outright boring however necessary they may be.
\end{abstract}

We describe the first stages of development of a safety training component for mainstream Chemistry courses that will allow students to identify safe and unsafe practices, undertake formal risk assessment, and enable them to improve the safety of their own environment. Our starting point is a Safety Training Workshop for our own 3rd year undergraduates, including case studies, a (competitive) team exercise (a lab 'scavenger hunt') and electronic self-testing, designed to alleviate the tedium. They also emphasise the cooperative nature of safe working and encourage teamwork - aspects which are normally neglected in degree programmes in Science. We also report on the positive student response.

\section{Introduction}

Good Laboratory Practice is not just a case of completing the paperwork and mentally ticking the 'safety box'. In a physical science subject where experimental research and observation are at the heart of the discipline, the skill set attained from risk assessment is a basic necessity. These skills are transferable but first require an innovative approach for their dissemination. In particular, one that is conducive to bringing about a change in mind-set for the average student.

Throughout Ireland and the UK there has been considerable infrastructure investment in the refurbishment and building of university laboratories. In Trinity College, the arrival in 2000 of new research and undergraduate teaching laboratories raised the profile of safety in our departmental activities. Safety requirements, constraints and protocols were of primary concern in the concept and design of these new facilities. This new compliance however had not percolated down into the conscience of the student body and this is the universal problem that we hoped to address through an interactive safety workshop.

\section{Course development}

Despite the effort exerted in safety training, students wait to be told rather than to think about safety information. To encourage our third year undergraduate students (current cohort of 62 students within a 4 year degree programme) to begin to put into practice the safety training they have received up to this point in their academic life, we designed a one-day intensive safety course. What we needed was a hands-on, problem-based course directed at advanced undergraduate level which could be incorporated into the third year laboratory programme. We began our course with a lab scavenger hunt and self test electronic quiz. The second half of the course involved lectures in safety awareness, with examination of some scenario based events to make students aware of potential lab hazards. Deriving key information from Material Safety Data Sheets (MSDS) and how to prepare informative and up-to-date Risk Assessments were discussed followed by a lecture given by an invited speaker from industry in order to show that the importance of being safety aware is not just a university matter but can have significant industrial consequences. 
Team exercises

One of the aims of the programme was to encourage critical thinking by our students in relation to good safety laboratory the groups' activities added to the enjoyment of the day and led to greater collaboration between students to identify all possible safety breaches in the laboratory.

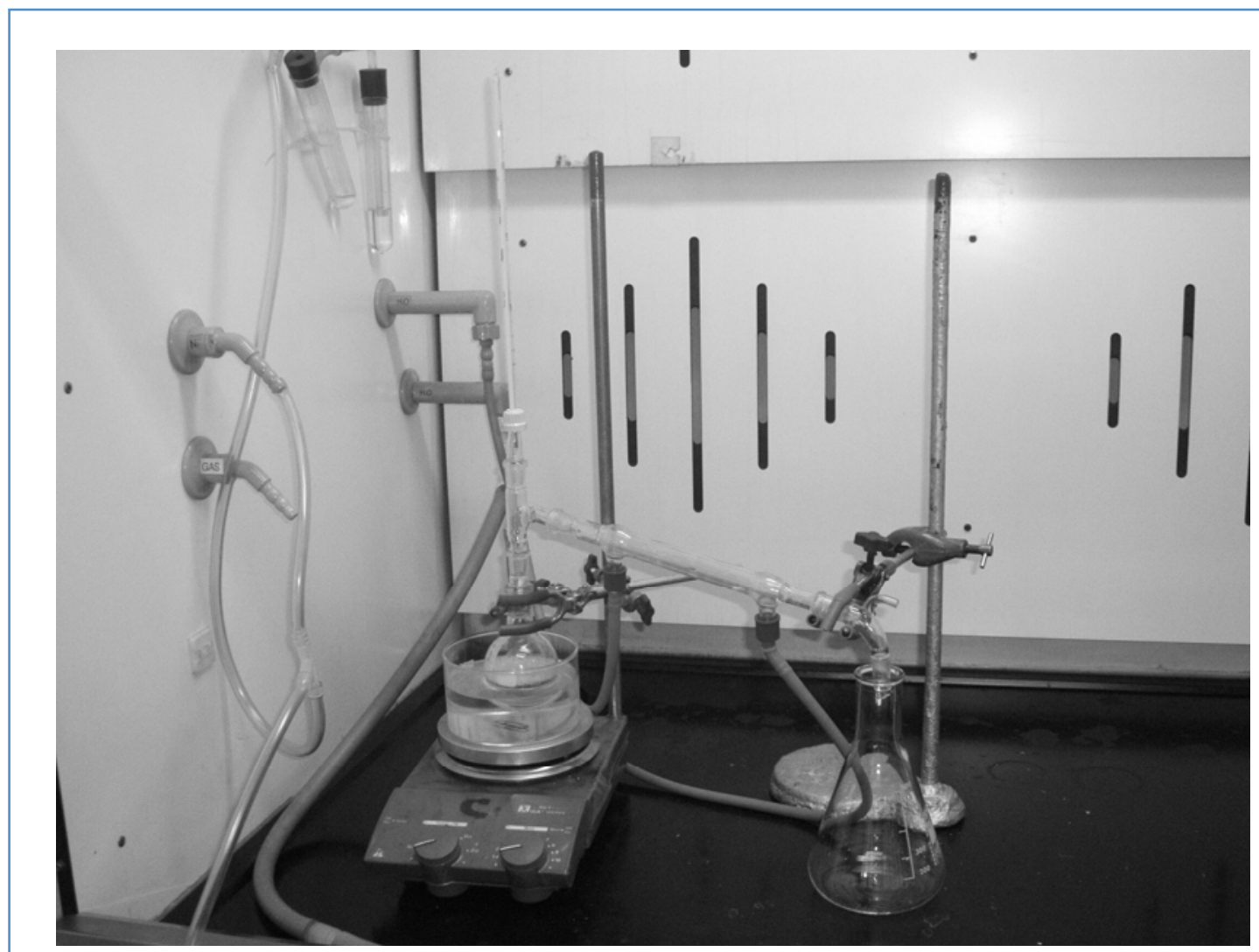

Electronic Self-test This was a computer based quiz, asking the students to answer 12 questions in 15 minutes. It was held at the start of the session to determine (or demonstrate) what the students did (and did not) already know, and to avoid the impression of it being an exam if held at the end. The questions covered both general matters (choice of fire extinguishers; identification of hazards and prioritisation of risks in experiments; choice of solvent) and more local matters (internal emergency phone number; assembly points etc). Scores ranged from $13 \%$ to $94 \%$ and the test was genuinely 'interactive' in that several of the questions (and our choice of 'correct' answers) provoked a

Figure 1: Scavenger hunt setup for distillation apparatus substantial and

practice. In order to promote safe-aware thinking, students were split into small groups and began the day with a laboratory activity. This team building exercise allowed students to examine the safety features of the chemistry undergraduate laboratory including the location of fire exits, fire blankets and extinguishers, first aid kits and eye wash stations. The first exercise was to fill in a map of the laboratory, marking the location of all safety features and equipment. The second part to the laboratory exercise took the form of a lab scavenger hunt. Several experiments were set up incorrectly around the laboratory by some of the postgraduate demonstrators and students were asked to spot potential safety hazards. Examples of experimental setups used were distillation, column and reflux apparatus (Figures 1 - 3). Some of the hazards included glassware not securely fitted, electrical cables crossing hot plates, water tubing not fitted correctly to condensers, etc. Students worked in groups of four to complete the exercise, which included looking through the laboratory for any other breaches of correct safety procedures, e.g. unlabelled solvent bottles, glassware clamped incorrectly, etc.

We found this interactive approach was vital to making students alive to safety-considerations in a positive way. The team who identified the most number of hazards in the laboratory were awarded a prize. This competitive element to sensible discussion from the students.

\section{Safety lectures and case studies}

Our afternoon lecture series began with a series of case studies designed to promote safety conscious thinking amongst students when it comes to protecting themselves against potential safety hazards. The lecture began with an examination of where our bodies are vulnerable to chemical attack and how chemicals may be absorbed by the body. Suitable clothing, such as laboratory coats, safety goggles and gloves were discussed. One case study used to illustrate the vital importance of protective equipment and the need to revisit and re-examine risk assessments of hazardous chemicals was that of Professor Karen Wetterhahn, who was poisoned by dimethylmercury, which seeped through the latex gloves she had been wearing. Even though Professor Wetterhahn followed all the standard safety procedures known at that time (use of labcoat, wearing safety goggles, working only in a fumehood and using latex gloves), the dose of mercury delivered to her body by one or two drops of dimethylmercury was enough to prove fatal. It was subsequently discovered that latex gloves did not provide sufficient protection against dimethylmercury and only heavy duty gloves should be used when handling this chemical. It was a wish of Professor Wetterhahn before her tragic death that her colleagues alert the scientific community to the 
dangers of dimethylmercury. Inclusion of this case study in our safety day workshop not only makes students aware of the real dangers of mercury poisoning but also of the importance of wearing suitable protective clothing and following up-to-date safety procedures.

Likely laboratory accidents were also discussed with some real-life cases considered, including explosions due to incorrect solvent disposal. These cases highlighted the need for students to review safety data sheets before attempting to dispose of solvent or chemical waste. They also brought home to students the medical, financial and research costs of accidents in university laboratories.

The lecture on MSDS and Risk assessment was designed as a first step towards preparing students to work within a legal framework of risk assessment. Greater emphasis was placed on the existence of such a framework, rather then on the details of current national (and rapidly changing) legislation. The talk covered: hazards and risks; chronic and acute responses;

contamination routes; getting information from labels, MSDS etc; and ended with the completion of our in-house risk assessment form for hazardous materials.

\section{Student feedback and conclusions}

The feedback we received from our undergraduate students was extremely positive, with most students commenting that their level of safety awareness had improved as a result of the safety workshop. The lab scavenger hunt was found to be one of the highlights of the day, with students noting they benefited from critically reviewing apparatus setups and would begin to do so with their own experimental setups. The team-building aspect of the exercise proved successful with some students remarking on the fact that they got to know their classmates
The last lecture of the series was given by a visitor from industry, Dr. Michael Gillen, a member of the Health and Safety Executive from IBEC, who spoke of the importance of 'walking the walk' and not just 'talking the talk' when it comes to chemical safety. The students heard more real life safety scenarios, this time from industry, which cemented the notion that being safety aware is a concern for anybody working in a laboratory environment.

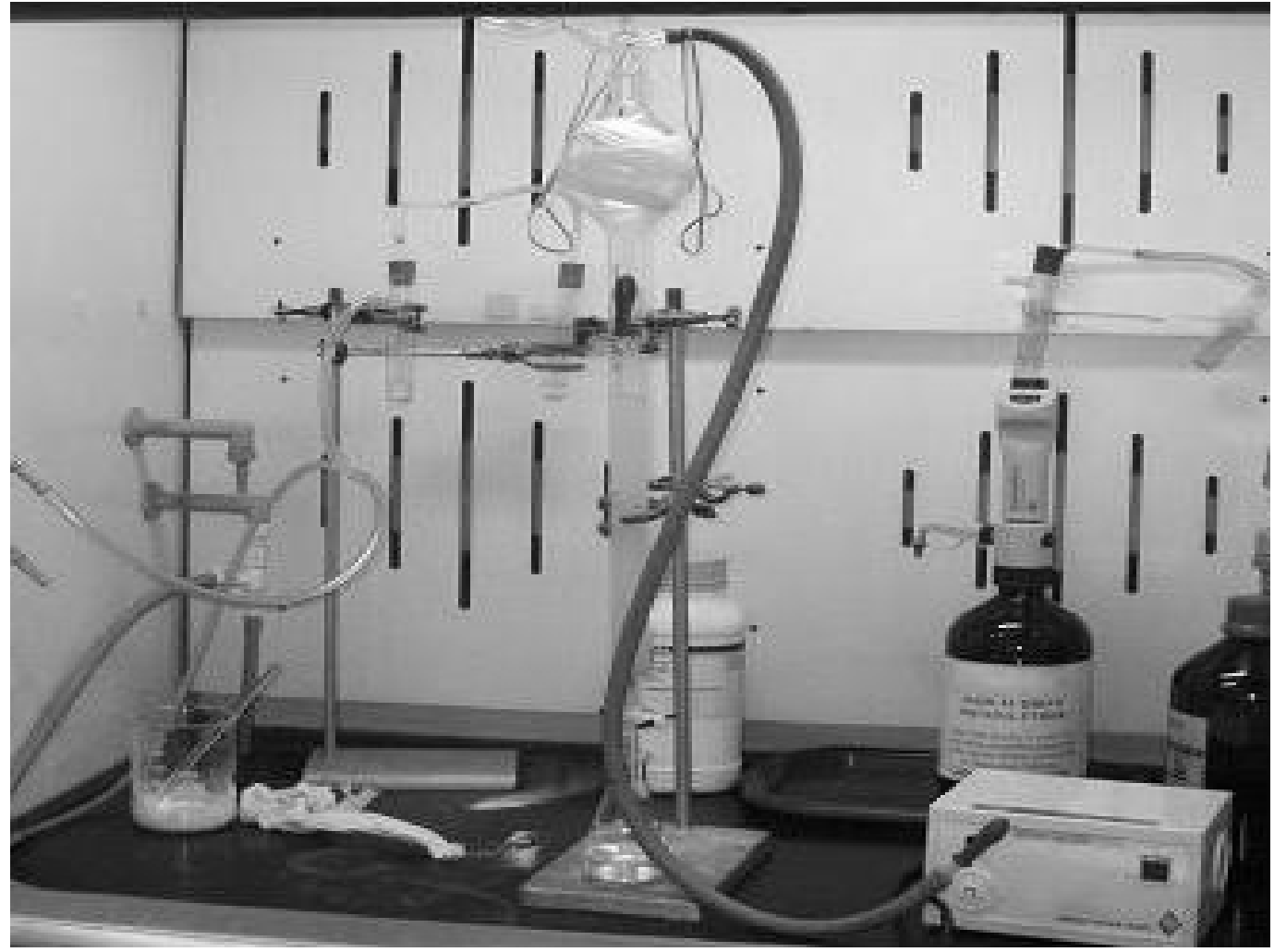

Figure 2: Scavenger hunt setup for column apparatus better as a result of working together. Some students also reported that the use of case studies made the message of safety awareness stick with them. One suggestion the students made for change in the future was the inclusion of an interactive safety workshop as part of the laboratory curriculum for each year of undergraduate study in Chemistry. Students felt that this could act as a beneficial revision course to revisit some good safety practices each year. The workshop in fact allowed us to identify some material suitable for use in similar exercises for our first and second year students and we will be incorporating a $2 \frac{1}{2}$ hour workshop into the practical course for both of these classes from 2007-8. Since entry to the degree programme in Chemistry at Trinity College Dublin is via a common Natural Science entry, dissemination of these activities and the corresponding contribution to the academic development of the students across the Science Faculty is ensured. In particular, the School of Chemistry plays a pivotal role in the teaching of (one) Physics and (one) Biochemistry-related undergraduate 
degree programmes: Physics and Chemistry of Advanced Materials and Medicinal Chemistry. Thus, the advances we have made by implementing this safety training programme will impact positively on these disciplines.

The implementation of the workshop with third year students has also allowed us to invite our final (fourth) year project students to join in our postgraduate safety training day, run jointly with the School of Biochemistry and Immunology at the beginning of each academic year. The impact of doing so was

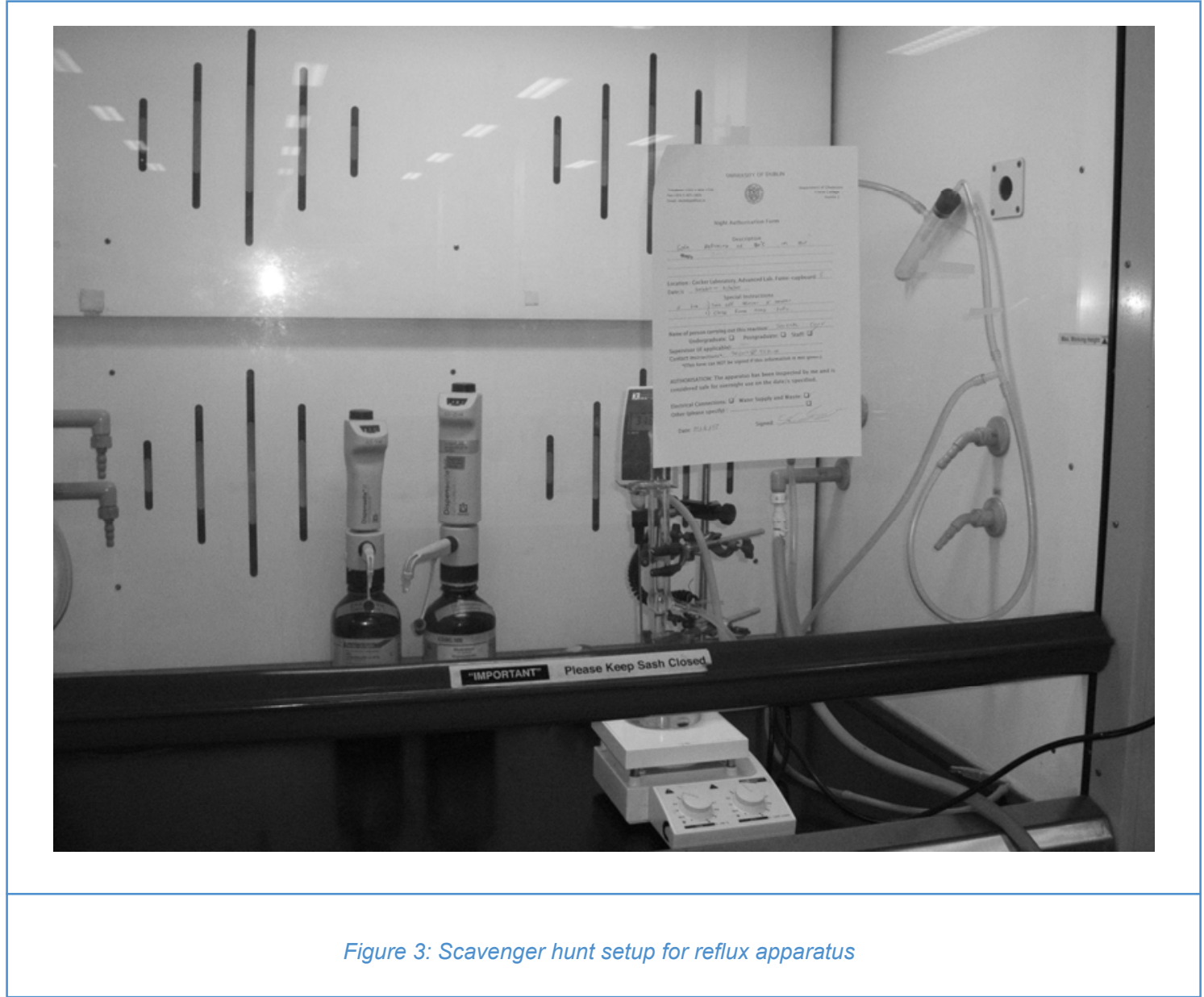

uniformly positive - the senior undergraduates were pleased (and flattered!) to be invited, and the inclusion of this group of students contributed to the social and educational aspects of the day. 